\title{
A Statistical Model of Respiratory Motion and Deformation of the Liver
}

\author{
J.M. Blackall, A.P. King, G.P. Penney, A. Adam, and D.J. Hawkes \\ Division of Radiological Sciences and Medical Engineering \\ The Guy's King's and St. Thomas' Schools of Medicine and Dentistry \\ Guy's Hospital, London SE1 9RT, UK
}

\begin{abstract}
This paper presents a statistical model of the respiratory motion and deformation of the liver. Individual models were made for seven volunteers using a series of MR images taken throughout the breathing cycle. One image was selected as a template and the others were registered to this using an intensity-based non-rigid registration algorithm. The resulting free-form transformations allowed us to map landmarks defined on the template image into their correct positions throughout the breathing cycle. Principal component analysis of these landmarks was used to produce a statistical model of motion and deformation. Results showing typical motion and deformation for a single volunteer are presented.
\end{abstract}

\section{Introduction}

Much of the motion and deformation of abdominal structures during interventional procedures may be attributed to respiration and so will be approximately periodic and potentially predictable over the breathing cycle. A number of studies have been carried out into the motion of the liver and other abdominal organs during respiration [1,2]. This paper describes a statistical approach to modelling breathing motion and deformation of abdominal structures.

\section{Method}

We construct volunteer-specific statistical models of the liver by using a voxel-based non-rigid registration technique [3] to coregister a number of MR images acquired throughout the breathing cycle. The resulting free-form transformations are used to propagate landmarks derived from a segmented liver surface of a template image to the other images [4]. The statistical analysis is performed using these corresponding surface landmarks to form a Point Distribution Model (PDM) as described in [5].

\section{Results}

Statistical models were constructed using the technique described above for a total of seven volunteers. In this section we present results from one of these which was selected as a representative example. 
Figure 1 illustrates the first mode of variation for the representative volunteer liver model. These renderings were produced by varying the weight for the first eigenmode only, whilst setting the others to zero. It can be seen that this mode of variation consists primarily of a rigid-body translation in the cranio-caudal direction. The magnitude of this translation, computed over all seven volunteers, is equal to $19 \pm 8 \mathrm{~mm}$ for shallow breathing, and $37 \pm 8 \mathrm{~mm}$ for deep breathing.

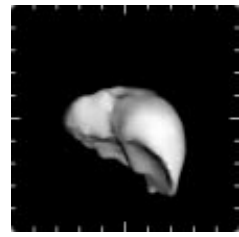

(a)

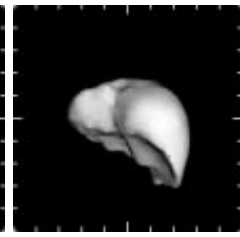

(b)

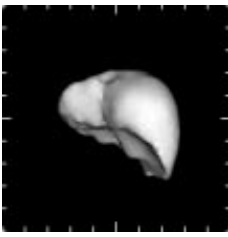

(c)

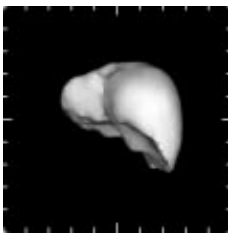

(d)

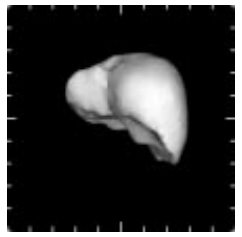

(e)

Fig. 1. Renderings illustrating the first mode of variation for a single volunteer. Animations of this and other modes of variation for all seven volunteers can be viewed at http://www-ipg.umds.ac.uk/MICCAI2001/liverModels.html

The other modes of variation typically capture deformation. The maximum magnitude of this deformation was approximately $15 \mathrm{~mm}$ for deep breathing and $10 \mathrm{~mm}$ for shallow breathing. A few areas seem more prone to large deformations, particularly the superior surface of the liver, which is in direct contact with the diaphragm, and the inferior surface of the liver, which is in contact with the back of the body cavity and is compressed against this surface as the diaphragm descends.

For all seven individuals the first eigenmode accounted for a large percentage of the total variation. For the volunteer shown in figure 1 the first five modes of variation accounted for $81 \%, 9.1 \%, 4 \%, 3.2 \%$ and $2.5 \%$ of the total variation respectively.

\section{Discussion}

This paper has presented work which applies a statistical approach to the analysis of respiratory motion and deformation in abdominal structures.

Previous work [4] has used binary segmented images to compute the non-rigid registrations. Our registrations were computed using the segmented grey level images, which allows the intensities of internal liver structures to contribute to the similarity measure, and may well lead to a more accurate registration. In [6] the entire MR images were coregistered, without segmentation of the liver. This approach will cause difficulties when there is a large relative motion between the liver and surrounding tissue.

Models of respiratory motion have the potential to widen the applicability of image guidance in the abdominal and thoracic region and could prove useful for treatment planning in other applications such as radiotherapy. 


\section{References}

1. S. C. Davies, A. L. Hill, R. B. Holmes et al. Ultrasound Quantitation of Respiratory Organ Motion in the Upper Abdomen. Br. J. Radiol., 67:1096-1102, 1994.

2. I. Suramo, M. Paivansalo, and V. Myllya. Cranio-caudal Movements of the Liver, Pancreas and Kidneys in Respiration. Acta Radiologica Diagnosis, 25(2):129-131, 1984.

3. D. Rueckert, C. Hayes, C. Studholme et al. Non-rigid Registration of Breast MR Images Using Mutual Information. Proc. MICCAI'98, Springer, 1144-1152, 1998.

4. A. F. Frangi, D. Rueckert, J. A. Schnabel et al. Automatic 3D ASM Construction via AtlasBased Landmarking and Volumetric Elastic Registration. In Proc. IPMI'01, Springer, 2001.

5. T. F. Cootes, C. J. Taylor, D. H. Cooper et al. Active Shape Models - Their Training and Application. Computer Vision and Image Understanding, 61(1):38-59, 1995.

6. T. Rohlfing, C. R. Maurer, Jr., W. G. O'Dell et al. Modeling Liver Motion and Deformation During the Respiratory Cycle using Intensity-Based Free-Form Registration of Gated MR Images. Proc. Medical Imaging 2000: Image Processing (SPIE 4322), 2001. 